\title{
Burkholderia cepacia complex infection in patients with cystic fibrosis
}

\author{
ESHWAR MAHENTHIRALINGAM, ADAM BALDWIN and PETER VANDAMME* \\ Cardiff School of Biosciences, Cardiff University, Cardiff, Wales, UK and * Laboratorium voor Microbiologie, \\ Faculteit Wetenschappen, Universiteit Gent, Ghent, Belgium
}

\begin{abstract}
The word 'complex' has several meanings and synonyms such as composite, obsession, heterogeneous, mixed and network, can all be used in its place. Our obsession with bacteria from the Burkholderia cepacia complex started in the early 1990s. In less than 10 years, we have seen the status of this bacterium move from: (i) a lesser known pseudomonad opportunist pathogen, (ii) to devastating infections transmitted between patients with cystic fibrosis (CF), (iii) through divisions into several new species, and (iv) now on towards one of the largest gram-negative genome sequencing projects. For microbiologists, hospital infection control officers, caregivers, and most of all the CF community, the changes in our understanding of the taxonomy, epidemiology and pathogenesis of the bacterium ' $B$. cepacia' are complex.
\end{abstract}

\section{The complex}

In 1992, the species Pseudomonas cepacia was reclassified as Burkholderia cepacia and it was assigned as the type species for the new genus Burkholderia [1]. Several new species were added to the genus in the following 5 years but it was not until 1997 that isolates classified as 'B. cepacia' were reexamined by polyphasic taxonomic approaches. Bacteria biochemically identified as B. cepacia were found to consist of at least five genetically distinct species or genomovars [2]. In hindsight, it was ironic that the species $B$. cepacia was designated as a reference for the genus [1] when its own taxonomy was still very uncertain. Further work has identified at least nine genomovars which constitute the B. cepacia complex; each of these is listed in Fig. 1. The taxonomy and identification of the $B$. cepacia complex have recently been reviewed in detail elsewhere [3] and the authors provided a summary of both biochemical and genetic means of genomovar identification. Six of the published genomovars have been assigned the following species names [3]: B. cepacia (the original specific epiphet is preserved for genomovar I), B. multivorans (formerly genomovar II), B. vietnamiensis (formerly genomovar V), B. stabilis (formerly genomovar IV) and B. ambifaria (formerly genomovar VII). B. cepacia genomovars III and VI await full species names if

Received 14 Feb. 2002; accepted 20 Feb. 2002.

Corresponding author: $\operatorname{Dr}$ E. Mahenthiralingam (e-mail: MahenthiralingamE@cardiff.ac.uk). simple differential tests can be found [3]. The description of genomovars VIII and IX is currently underway (P. Vandamme and E. Mahenthiralingam, unpublished data) (Fig. 1). The name ' $B$. anthina' has been proposed for strains of genomovar VIII, as a number of different tests enabling the identification of this species have been found (P. Vandamme and E. Mahenthiralingam, unpublished data). The reference strain for genomovar IX, LMG 14191, had already been formerly named as the species B. pyrrocinia [2]. It is now clear from polyphasic taxonomic approaches that this strain and other closely related isolates constitute a distinct genomovar within the current B. cepacia complex (Fig. 1) (P. Vandamme and E. Mahenthiralingam, unpublished data). To assist researchers and microbiologists studying B. cepacia complex bacteria, a panel of strains representative of the first five genomovars was published 2 years ago [4]. With the identification of four further genomovars this useful strain panel is already in need of updating and expansion.

\section{Composite genomovar tests and identification of genomovars based on the $\operatorname{rec} A$ gene}

The $B$. cepacia complex genomovars are very closely related, with few if any biochemical reactions able to separate them and multiple tests often required for accurate identification [3]. While seeking rapid molecular tests for identification of $B$. cepacia complex bacteria, we discovered that there was sufficient 

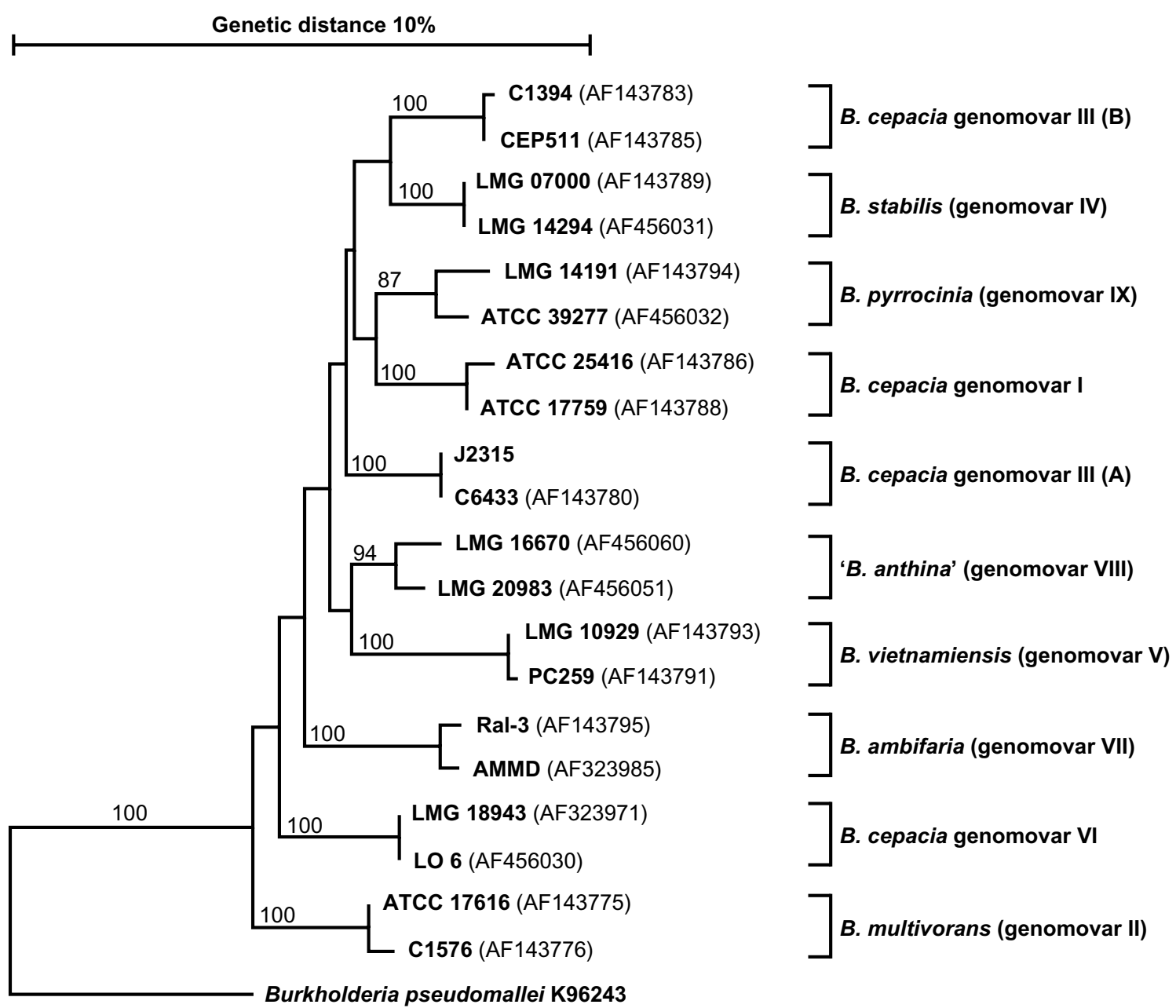

Fig. 1. A phylogenetic tree of $r e c A$ gene sequences indicating the presence of nine B. cepacia complex genomovars. Nucleotide sequences were determined, aligned and used to construct the neighbour joining tree as described previously [5]. The strains representative of each genomovar are indicated and the GenBank accession no. for published and novel rec $A$ gene sequences is provided in brackets. Sequence data for the B. pseudomallei strain K96243 and B. cepacia genomovar III strain J2315 recA genes were generated by the Pathogen Sequencing Unit at the Sanger Institute, and can be obtained from http://www.sangr.ac.uk/ Projects/Microbes. The genomovar or species name for each distinct cluster is indicated on the right. Genetic distance and bootstrap values $>70 \%$ for each node are shown.

variation in nucleotide sequence of the $r e c A$ gene to enable discrimination of the first five genomovars described [5]. We have subsequently found that analysis of recA sequence variation, in general, correlates well with the genomovar taxonomy. Restriction fragment length polymorphism (RFLP) analysis of the rec $A$ gene can serve as a primary means of identifying taxonomic diversity among isolates [5] and $>50 \mathrm{~B}$. cepacia complex RFLP types have now been found when the gene is cut with the restriction enzyme HaeIII (E. Mahenthiralingam and P. Vandamme, unpublished data). Novel recA gene RFLP types that do not correlate with a known genomovar can then be subjected to nucleotide sequence analysis to enable phylogenetic predication of genomovar status $[3,5]$.

Phylogenetic analysis of recA gene sequences from strains representative of all nine current $B$. cepacia complex genomovars is shown in Fig. 1. All nine $B$. cepacia complex genomovars separate into distinct arms of the phylogenetic tree. Interestingly, sequence variation in the recA gene also appears to separate strains of genomovar III into two distinct clusters: III-A and III-B (Fig. 1); the prevalence of each lineage of genomovar III varies between different CF populations (see below). A similar subdivision of $B$. cepacia genomovar I strains (defined by polyphasic taxonomic analysis) into two distinct $r e c A$ phylogenetic clusters has also been observed (P. Vandamme and $E$. Mahenthiralingam, unpublished data).

The PCR primers, BCR1 and BCR2, originally designed to amplify the full length $r e c A$ gene remain highly specific for $B$. cepacia complex bacteria and do not cross-react with closely related Burkholderia spp. or other common CF pathogens such as $P$. aeruginosa [5]. This specificity has led to the successful application of recA PCR directly to CF sputum [6]. Such direct testing for suspected $B$. cepacia complex infection may prove very useful in hospital infection control and the clinical management of $\mathrm{CF}$ patients. Other genomovar-specific recA-based PCR tests [5] appear to be less specific in light of the identification of further taxonomic diversity. For example, the PCR 
primers designed to be specific for $B$. cepacia genomovar I [5] cross-react with B. pyrrocinia and fail to detect some genomovar I isolates which fall into the second recA genomovar I cluster mentioned above (E. Mahenthiralingam, unpublished data). Hence, even with molecular genomovar identification, multiple tests (RFLP, sequence analysis and genomovar-specific PCR) may be required, with no single diagnostic approach possessing absolute specificity.

\section{Genomovar prevalence in $\mathrm{CF}$ infection}

From the initial description of five genomovars within the $B$. cepacia complex, it was clear that strains from each genomovar may cause infection in patients with CF [2]. This basic pathogenic trait has continued to be true for all further genomovars described and strains from $\mathrm{CF}$ infection may be found in each evolutionary arm of the diverse $B$. cepacia complex phylogenetic tree (Fig. 1), (E. Mahenthiralingam and P. Vandamme, unpublished data). Because of the development of rapid and widely applicable genomovar identification tests, national systematic analysis of the prevalence of each B. cepacia complex species has now been examined in the USA [7] and Canada [8]. A smaller study examining the prevalence and epidemiology of $B$. cepacia complex bacteria among CF patients attending four treatment centres in Italy has also been published [9]. A summary of the findings of the latter studies is presented in Table 1 [7-9]. Studies based on the examination of collections of $B$. cepacia complex isolates had indicated that genomovar III and $B$. multivorans were the predominant $\mathrm{CF}$ pathogens $[2,5]$. These findings have been validated by the systematic studies of prevalence in CF (Table 1).

B. cepacia genomovar III is the most prevalent genomovar in $\mathrm{CF}$, causing $>50 \%$ of $\mathrm{CF}$ infections in all CF populations examined (Table 1). Interestingly, if strains of genomovar III are divided into their separate recA lineages of III-A and III-B (see Fig. 1), distinct differences between the USA, Canadian and Italian CF populations can be observed. Within the USA, strains of B. cepacia genomovar III-B represent $75 \%$ of genomovar III infections [7], whereas in Canada and the four Italian CF centres examined, III-A strains were dominant, accounting for $>70 \%$ of genomovar III infections in each population (Table 1) $[8,9]$. The basis for these distinct differences is unknown as yet.

B. multivorans is the second most predominant $\mathrm{CF}$ pathogen after B. cepacia genomovar III (Table 1). Once again though, there were differences between the three CF populations examined. In the USA, $B$. multivorans CF infection occurs almost as widely as genomovar III infection (38\% of cases) [7]. In Canada and Italy, B. multivorans caused $\leqslant 10 \%$ of $B$. cepacia complex infections (Table 1) $[8,9]$. The prevalence of all the remaining $B$. cepacia genomovars in $\mathrm{CF}$ was at
Table 1. The prevalence of B. cepacia complex genomovars among three $\mathrm{CF}$ populations

\begin{tabular}{lcccc}
\hline & \multicolumn{3}{c}{ Percentage prevalence } & \\
\cline { 2 - 4 } Genomovar or & $\begin{array}{c}\text { USA }^{*} \\
(606\end{array}$ & $\begin{array}{c}\text { Canada }^{\dagger} \\
(475\end{array}$ & $\begin{array}{c}\text { Italy } \\
(59\end{array}$ & \\
patients $)$ & patients $)$ & patients $)$ & Mean \\
\hline Genomovar I & 2.6 & 0.2 & 4.8 & 2.5 \\
B. multivorans & 37.8 & 9.3 & 4.8 & 17.3 \\
Genomovar III & 50.0 & 80.0 & 72.6 & 67.5 \\
B. stabilis & 0.2 & 3.8 & 3.2 & 2.4 \\
B. vietnamiensis & 5.1 & 1.6 & 0 & 2.2 \\
Genomovar VI & 2.0 & 0 & 0 & 0.7 \\
Genomovar VII & 0.7 & 0 & 0 & 0.2 \\
Indeterminate & 1.6 & 1.8 & 14.5 & 5.9 \\
\hline
\end{tabular}

* Data from LiPuma et al. (2001) [7].

${ }^{\dagger}$ Data from Speert et al. (2002) [8].

tData adapted from Agodi et al. (2001) [9] and representative of 59 patients attending four $\mathrm{CF}$ treatment centres.

most $5 \%$ in the populations examined (Table 1). Hence, taxonomic classification of predominant $\mathrm{CF}$ species appears nearly complete, with $B$. cepacia genomovar III and B. multivorans accounting for $95 \%$ of the infections (Table 1). So, which species are the most problematic from a clinical standpoint?

\section{Molecular epidemiology}

Spread of respiratory infections in patients with CF, although a controversial area of research, had not been a significant clinical problem until the early 1990s. Reports in the UK and USA indicated that $P$. cepacia, as it was known then, was capable of nosocomial transmission [10,11]. In addition to risk of spread, infection was also linked to a rapid decline in clinical condition in certain CF patients, which became known as ' $B$. cepacia' syndrome. These hazards resulted in the cohorting of CF patients colonised with B. cepacia in many treatment centres.

During the early 1990s concern about the increasing incidence of B. cepacia-infected patients at a treatment centre in Vancouver, Canada, led to the application of random amplified polymorphic DNA (RAPD) fingerprinting to the epidemiological analysis of ' $B$. cepacia' infection [12]. Several B. cepacia strain types were each found to infect multiple patients [12]. Moreover, epidemiological observations suggested that transmission had been due to direct patient-to-patient contact both within and outside the hospital setting, as had been observed in earlier studies [9]. One of the strains encountered in the Vancouver $\mathrm{CF}$ patient population was designated as RAPD strain type 2 [12]. This strain type was a member of a clonal lineage of strains which first infected $\mathrm{CF}$ patients in Toronto, subsequently spread across Canada and was also introduced into the UK CF population, probably as a result of patient contact during CF summer camps [13]. This highly infectious strain had at the time been recently identified 
and was known as the ET12 (electrophoretic type 12) [14] or cable pilus-encoding strain [13].

Our success with the RAPD typing technique was not just limited to accurate molecular epidemiological analysis. We were also able to identify a very useful DNA marker, the B. cepacia epidemic strain marker (BCESM) [15]. The BCESM DNA was associated with B. cepacia strains that were known to have spread among CF patients in Vancouver and other parts of Canada [15]. Hence it could be used as a rapid diagnostic marker to alert $\mathrm{CF}$ patients and clinicians to the potential risks associated with infection by these strains. By examining the genomovar status of BCESM-positive isolates, we now understand that this marker is found exclusively in strains of B. cepacia genomovar III [5], but is not carried by all strains. Among Canadian CF patients, BCESM-positive strains account for $>80 \%$ of all genomovar III infections [8]. Several of these strain types, not just the dominant ET12 strain, were capable of epidemic spread among $\mathrm{CF}$ patients $[5,12]$. Within the USA, the picture in terms of incidence of BCESM-encoding strains is very different, with positive strains accounting for only $23 \%$ of all B. cepacia genomovar III strains encountered [7].

The BCESM region of the genome is unstable, particularly in strains of genomovar III-B, and can be lost after passage in vitro (E. Mahenthiralingam, unpublished data). In the USA, genomovar III-B strains most frequently lacked the marker (64\% negative) and were the predominant genomovar III strain lineage encountered (Table 1) [7]. The BCESM DNA is much more stable in B. cepacia genomovar III-A strains [5], suggesting that they may be the 'natural' hosts for this unusual genomic DNA element. The instability of the BCESM element, its size and the potential virulence genes encoded within this region are currently under investigation (E. Mahenthiralingam and A. Baldwin, unpublished data). Overall, while it is clear that BCESM DNA is not an absolute marker of the ability to cause infection or spread among CF patients [7], in CF populations where BCESM-positive strains dominate, they have proved to be highly virulent and very problematic [16; see below].

Although B. cepacia genomovar III strains have been implicated in the majority of the published accounts of patient-to-patient spread in CF [4], the ability of all the other genomovars to cause outbreaks of infection cannot be ignored. A common phenotypic trait of all $B$. cepacia complex bacteria is their intrinsic resistance to multiple antibiotics. B. cepacia complex bacteria appear to share the same ability for nosocomial spread as other drug-resistant bacterial infections such as methicillin-resistant Staphylococcus aureus and vancomycin-resistant enterococci. Outbreaks of B. multivorans infection, affecting large numbers of patients, have been reported in the UK [17] and France [18]. Current UK infection control guidelines recommend individual segregation of $\mathrm{CF}$ patients infected with $B$. cepacia complex bacteria; because of the mixed epidemiology which has been observed with $B$. cepacia complex bacteria, these strict measures are the best current means of preventing strain transmission.

\section{Clinical outcome in relation to genomovar}

Few studies have systematically examined clinical outcome in relation to B. cepacia complex genomovar. Strains isolated from $C F$ patients attending the Vancouver treatment centres had been collected since 1981 and this enabled a retrospective examination of epidemiology and clinical outcome to be determined for this CF patient population [16]). Initial infection control procedures implemented at the $\mathrm{CF}$ clinics involved cohorting of all ' $B$. cepacia'-positive patients, and their separation from $P$. aeruginosa-positive and non-colonised CF patients. The B. cepacia genomovar status of the Vancouver CF population was heterogeneous. Strains of genomovar III-A (four different strain types, including ET12) accounted for the majority of cases of infection (46 of 52 patients). Distinct B. multivorans strains were isolated from 19 of 62 patients, and the remaining three patients were infected with B. stabilis, B. vietnamiensis and an indeterminate $B$. cepacia complex isolate, respectively [16].

Epidemiology and clinical outcome associated with $B$. cepacia genomovar III and B. multivorans infection were strikingly different [16]). Patient-to-patient spread of four different genomovar III-A strains (each encoding the BCESM) had occurred until the introduction of new infection control procedures (individual segregation of $B$. cepacia complex-infected patients) in 1995. No spread of B. multivorans strains (apart from transient strain-sharing between two $\mathrm{CF}$ siblings) was observed during the entire 17 years of study. Genomovar III infections were more likely to be chronic, whereas the majority of cases of $B$. multivorans infection were transient. Patients with genomovar III infection suffered the greatest mortality (20 of 46 patients died), but only three of the $19 \mathrm{~B}$. multivoransinfected patients died (notably two of these were cocolonised with genomovar III at the time of death). From an infection control stance, the most worrying feature of $B$. cepacia genomovar III infection was the ability of these strains to replace infection with $B$. multivorans, which occurred in six cases of infection. However, with the introduction of segregation of B. cepacia complex-infected patients as a result of the strain-typing observations, spread of infection and the incidence of B. cepacia genomovar III infection were significantly reduced after 1995 [16].

Is the Vancouver experience representative of what may happen with $B$. cepacia complex infection in patients with CF? Experience gained from lung transplantation 
of $B$. cepacia complex-infected CF patients also shows that infection with genomovar III is associated with significant postoperative mortality, whereas infection by other genomovars is less problematic $[19,20]$. These findings correlate with the greater mortality linked to B. cepacia genomovar III infection in Vancouver [16]. However, the absence of spread and virulence of $B$. multivorans is not shown by other studies [17, 18]. Significant transmission was observed during an outbreak of infection with a $B$. multivorans strain among Glasgow CF patients [17]. Fatal B. multivorans septicaemias and multiple patients infected with single strains have been observed in French CF patients [18]. Phylogenetic comparison of the Glasgow B. mutivorans strain (represented by strain C1576) indicates that it is from a different strain lineage when compared with the soil-isolated reference strain ATCC 17616, which is representative of the same $\operatorname{rec} A$ lineage of $B$. multivorans strains encountered in Vancouver (see Fig. 1). Further study may uncover whether there are discrete epidemiological differences among $B$. multivorans strains that are similar to those observed for BCESMpositive B. cepacia genomovar III strains. Overall, although infection with $B$. cepacia genomovar III bacteria represents a significant clinical risk to patients with $\mathrm{CF}$ [16], B. multivorans and all the remaining genomovars are capable of causing devastating infections within any given patient.

\section{B. cepacia complex virulence}

Virulence in CF, perhaps more than any other area of $B$. cepacia biology, is still open to question and further research. What are the specific bacterial factors that lead to pathogenesis? Why do some patients succumb rapidly to infection while others remain healthy, despite being infected with the same strain of B. cepacia? Why does the outcome and prevalence of infection vary with B. cepacia genomovar and $\mathrm{CF}$ patient population? $B$. cepacia complex bacteria possess many of the bacterial factors known to play a role in $P$. aeruginosa $\mathrm{CF}$ infection [21]. Many of the answers to virulence lie within the fact that all B. cepacia complex bacteria have very large and unusual genomes [5,22]. Average genome size is $c .8 \mathrm{Mb}$ and the DNA is carried on at least two, or frequently three or four large replicons $[5,22]$. Hence the potential for strain-to-strain variation in genomic content is enormous and perhaps contributes to the heterogeneity in virulence and pathogenesis. The B. cepacia genomovar III strain, J2315 (LMG16656), was recently chosen as the subject of genome sequence analysis (http://www.sanger.ac.uk/ Projects/B_cepacia/). Strain J2315 was the index strain associated with spread of the ET12 cable pilus-positive strain among CF patients in Edinburgh [10]. The genomovar III-A CF strain lineage it represents (Fig. 1) is unique in being the only strain to date that possesses both the cable pilus gene and BCESM [5]. Strain J2315 and other clonal isolates (e.g., K56-2 or BC7) are amenable to various types of genetic manipulation [4] and, in combination with the genome sequence, these tools should provide an excellent means to uncover virulence factors within the $B$. cepacia complex.

\section{An obsessed network}

One group, the International Burkholderia cepacia Working Group (IBCWG; http://allserv.rug.ac.be/ $\sim$ ppvandam/cepacia/) has contributed considerably to our understanding of this group of micro-organisms. The IBCWG was created informally in 1995 after a small meeting in Washington, DC, where the focus was specifically on B. cepacia infection in patients with CF. The group now has annual meetings with around 50 participants and research within the network covers all aspects of $B$. cepacia biology, from clinical through ecological and industrial. With this broad base of knowledge and expertise, further explanations of the virulence and pathogenesis of the B. cepacia complex in patients with $\mathrm{CF}$ will be forthcoming.

We are indebted to the United Kingdom Cystic Fibrosis Trust (E.M., grant nos PJ472 and PJ501) and the Fund for Scientific Research Flanders (P.V.) for financial support. We thank the collaborative efforts of investigators within the IBCWG for assistance with our research on the B. cepacia complex.

\section{References}

1. Yabuuchi E, Kosako Y, Oyaizu $\mathrm{H}$ et al. Proposal of Burkholderia gen. nov. and transfer of seven species of the genus Pseudomonas homology group II to the new genus, with the type species Burkholderia cepacia (Palleroni and Holmes 1981) comb. nov. Microbiol Immunol 1992; 36: 1251-1275.

2. Vandamme P, Holmes B, Vancanneyt M et al. Occurrence of multiple genomovars of Burkholderia cepacia in cystic fibrosis patients and proposal of Burkholderia multivorans sp. nov. Int J Syst Bacteriol 1997; 47: 1188-1200.

3. Coenye T, Vandamme P, Govan JRW, LiPuma JJ. Taxonomy and identification of the Burkholderia cepacia complex. J Clin Microbiol 2001; 39: 3427-3436.

4. Mahenthiralingam E, Coenye T, Chung JW et al. Diagnostically and experimentally useful panel of strains from the Burkholderia cepacia complex. J Clin Microbiol 2000; 38: 910-913.

5. Mahenthiralingam E, Bischof J, Byrne SK et al. DNA-based diagnostic approaches for identification of Burkholderia cepacia complex, Burkholderia vietnamiensis, Burkholderia multivorans, Burkholderia stabilis, and Burkholderia cepacia genomovars I and III. J Clin Microbiol 2000; 38: 3165-3173.

6. McDowell A, Mahenthiralingam E, Moore JE et al. PCR-based detection and identification of Burkholderia cepacia complex pathogens in sputum from cystic fibrosis patients. $J$ Clin Microbiol 2001; 39: 4247-4255.

7. LiPuma JJ, Spilker T, Gill LH, Campbell PW, Liu L, Mahenthiralingam E. Disproportionate distribution of Burkholderia cepacia complex species and transmissibility markers in cystic fibrosis. Am J Respir Crit Care Med 2001; 164: 92-96.

8. Speert DP, Henry D, Vandamme P, Corey M, Mahenthiralingam E. Epidemiology of Burkholderia cepacia complex in patients with cystic fibrosis in Canada: geographical distribution and clustering of strains. Emerg Infect Dis 2002; 8 (in press).

9. Agodi A, Mahenthiralingam E, Barchitta M, Giannino V, Sciacca A, Stefani S. Burkholderia cepacia complex infection in Italian patients with cystic fibrosis: prevalence, epidemiology, and genomovar status. J Clin Microbiol 2001; 39: 2891-2896. 
10. Govan JRW, Brown PH, Maddison $\mathrm{J}$ et al. Evidence for transmission of Pseudomonas cepacia by social contact in cystic fibrosis. Lancet 1993; 342: 15-19.

11. LiPuma JJ. Burkholderia cepacia epidemiology and pathogenesis: implications for infection control. Curr Opin Pulm Med 1998; 4: 337-341.

12. Mahenthiralingam E, Campbell ME, Henry DA, Speert DP Epidemiology of Burkholderia cepacia infection in patients with cystic fibrosis: analysis by randomly amplified polymorphic DNA fingerprinting. J Clin Microbiol 1996; 34: 2914-2920.

13. Sun L, Jiang RZ, Steinbach S et al. The emergence of a highly transmissible lineage of cbl+ Pseudomonas (Burkholderia) cepacia causing CF centre epidemics in North America and Britain. Nat Med 1995; 1: 661-666.

14. Johnson WM, Tyler SD, Rozee KR. Linkage analysis of geographic and clinical clusters in Pseudomonas cepacia infections by multilocus enzyme electrophoresis and ribotyping. J Clin Microbiol 1994; 32: 924-930.

15. Mahenthiralingam E, Simpson DA, Speert DP. Identification and characterization of a novel DNA marker associated with epidemic Burkholderia cepacia strains recovered from patients with cystic fibrosis. J Clin Microbiol 1997; 35: 808-816.

16. Mahenthiralingam E, Vandamme $\mathrm{P}$, Campbell $\mathrm{ME}$ et al. Infection with Burkholderia cepacia complex genomovars in patients with cystic fibrosis: virulent transmissible strains of genomovar III can replace Burkholderia multivorans. Clin Infect Dis 2001; 33: 1469-1475.

17. Whiteford ML, Wilkinson JD, McColl JH et al. Outcome of Burkholderia (Pseudomonas) cepacia colonisation in children with cystic fibrosis following a hospital outbreak. Thorax 1995; 50: 1194-1198.

18. Segonds C, Heulin T, Marty N, Chabanon G. Differentiation of Burkholderia species by PCR-restriction fragment length polymorphism analysis of the 16S rRNA gene and application to cystic fibrosis isolates. J Clin Microbiol 1999; 37: 2201-2208.

19. Aris RM, Routh JC, LiPuma JJ, Heath DG, Gilligan PH. Lung transplantation for cystic fibrosis patients with Burkholderia cepacia complex. Survival linked to genomovar type. Am J Respir Crit Care Med 2001; 164, 2102-2106.

20. De Soyza A, McDowell A, Archer L et al. Burkholderia cepacia complex genomovars and pulmonary transplantation outcomes in patients with cystic fibrosis. Lancet 2001; 358: $1780-1781$.

21. Govan JRW, Deretic V. Microbial pathogenesis in cystic fibrosis: mucoid Pseudomonas aeruginosa and Burkholderia cepacia. Microbiol Rev 1996; 60, 539-574.

22. Lessie TG, Hendrickson W, Manning BD, Devereux R. Genomic complexity and plasticity of Burkholderia cepacia. FEMS Microbiol Lett 1996; 144: 117-128. 